\title{
Les agriculteurs et la pollution des eaux. Proposition d'une géographie des pratiques
}

\author{
Christophe Toussaint Soulard \\ Géographe, INRA-SAD, LISTO, BP 87999, 21079 Dijon cedex, France
}

Cet article est un bon exemple des travaux que nous cherchons à encourager dans la revue Natures Sciences sociétés. L'auteur y aborde une question d'environnement : la pollution des eaux par les nitrates, en liant la représentation que s'en font prescripteurs et praticiens à la matérialité des faits que constituent les pratiques de fertilisation azotée dans leurs dimensions spatiotemporelles. À partir de l'étude d'un cas concret, il montre ce qu'une géographie des pratiques peut apporter à la résolution de cette question.

La Rédaction

\section{Mots-clés :}

pollution de l'eau ; fertilisation azotée ; pratiques des agriculteurs; géographie; Bourgogne

\section{Keywords:} water pollution; nitrates; farmers; spatial practices; geography; Burgundy

\begin{abstract}
Résumé - Cet article propose d'étudier la prise en compte des problèmes de pollution des eaux en agriculture. L'étude des relations à l'espace des agriculteurs sert de fil conducteur à une recherche de terrain conduite dans une petite région du département de l'Yonne. L'analyse est centrée sur l'étude de l'ajustement spatiotemporel de la fertilisation azotée. Elle repose sur une confrontation des écarts entre le modèle technique des recommandations, fondé sur le bilan prévisionnel de l'azote, et le modèle pratique des agriculteurs. Les résultats révèlent que les catégories spatiotemporelles des agriculteurs diffèrent de celles des recommandations. Cet écart est ensuite analysé sous l'angle de ce qu'il signifie du point de vue des capacités des agriculteurs à intégrer le risque de pollution dans leurs pratiques. Trois échelles d'observations sont alors mobilisées : l'exploitation agricole ; l'unité sols à risque ; le groupe de parcelles situées sur ces sols. Les résultats permettent de porter un diagnostic de géographie des pratiques sur les marges de manœuvre qu'auraient les agriculteurs pour réorienter leurs pratiques vers le respect de l'environnement.
\end{abstract}

\begin{abstract}
Farmers and water pollution by nitrates. A geographical approach to agricultural practices. The paper analyses how farmers take water pollution by nitrates into account. The author proposes a geographical approach to farmer practices aimed at confronting the farmers' values and spatial practices with the spatial delimitations of other actors concerned by a local pollution issue. The research methodology involved surveys of twenty farmers in a French rural area (Yonne department) who were compelled to take a local problem of nitrogen pollution into account in their farming practice. Two spatial entities were combined in studying changes in their practices: the farm and the catchment area. The perception of space by the farmers relates to "the farm lands" and to "individual fields or groups of fields" on their farms. These spatial categories differ from the ones used by agricultural advisers and regulations to circumscribe vulnerable areas and land units. The study of field by field adjustment of nitrogen inputs on wheat shows a diversity of space delimitations among farmers. Mapping these results allows the identification of the spatial adjustments and change of practice imposed by the consideration of pollution risks. In conclusion, these results allow a reflection on the relationships between environmental values and environment-related practices.
\end{abstract}

Auteur correspondant : christophe.soulard@enesad.inra.fr 


\section{Introduction : l'agriculture et l'environnement en géographie}

Parce qu'ils se situent à l'interface entre la nature et la culture, entre le milieu et la société, entre les sciences sociales et naturelles, les problèmes d'environnement sont particulièrement délicats à appréhender par le chercheur (Jollivet et Pavé, 1993). Il est d'autant plus difficile de s'y attaquer seul. Et la tentation est alors grande, soit de donner à l'environnement naturel une réalité en soi, alors indépendante du social, soit de le réduire au seul produit d'une construction sociale ou politique. Pour éviter ces écueils, considérer l'environnement comme " problème » ou «question » posée par la société à propos d'un phénomène naturel que la société perturbe est alors une alternative possible. Il s'agit dès lors de voir dans l'environnement, non pas un objet en soi, mais un processus qu'il est susceptible d'éclairer suivant une certaine dimension d'analyse qui prenne en compte et confronte des faits naturels, des points de vue sociaux, des savoirs multiples, des moyens d'action et des dispositifs divers, au travers desquels l'environnement se constitue en problème pour certains acteurs, autour d'un fait de nature donné, localisé dans l'espace et daté dans le temps.

Une telle perspective constructiviste nous paraît tout à fait utile pour spécifier un point de vue possible de la géographie sur l'environnement dans la perspective du développement durable (Robic et Mathieu, 2001). En effet, dans la perspective d'une "géographie recentrée », ou "traversière », que plusieurs auteurs appellent de leurs vœux (Mathieu, 1992 ; Pinchemel, 1995; Bertrand, 2002), étudier de façon dialectique l'articulation milieu-société via l'espace nous semble une voie féconde pour penser, sans les séparer, la configuration matérielle et le contenu social d'un problème d'environnement. C'est ce que nous avons tenté de faire en analysant un problème d'environnement dont l'origine est attribuée principalement à l'agriculture : la pollution des eaux souterraines par les nitrates.

Parce qu'elle est invisible et peu facile à circonscrire dans l'espace et dans le temps, on peut se demander comment la pollution diffuse par les nitrates peut se constituer en un problème circonscrit dans l'espace. Mais on peut également faire l'hypothèse que ce cas de figure offre justement un bon exemple de l'effort qu'ont à produire les acteurs pour localiser ce type de problème et imaginer des solutions pertinentes et acceptables. Or, cette difficulté suggère pour les géographes des pistes $\mathrm{d}$ 'investigations multiples. Celle que nous avons retenue propose d'ériger en objet d'étude le rapport à l'espace, au milieu et au territoire des agriculteurs. Dans cette perspective, notre hypothèse de travail sera que l'environnement génère des changements techniques qu'il convient d'analyser dans les ajustements spatiaux qu'ils suscitent. Pour y parvenir, nous privilégierons l'analyse des écarts entre les recommandations techniques de préservation de la qualité de l'eau et les pratiques qu'elles mettent en cause.

Nous nous rapprocherons ainsi des travaux situés à l'interface entre l'agronomie et la géographie, notamment ceux qui abordent l'exploitation agricole et le territoire d'action environnementale comme des lieux de différenciations et de coordinations spatiales des pratiques (Deffontaines et Brossier, 2000; Papy, 2001). Mais nous nous inspirerons aussi des recherches situées à l'interface entre la géographie et les sciences sociales, et qui prennent comme objet d'étude, soit les confrontations entre savoirs savants et vernaculaires dans la gestion écologique des milieux (Blanc-Pamard, 1986), soit les agencements spatiaux qui accompagnent l'inscription dans le territoire des normes et des actions environnementales (Rémy et al., 2004).

C'est ce que nous résumons en parlant d'une "géographie des pratiques », dont le projet est d'identifier et de confronter les catégories spatiales et temporelles que les acteurs mobilisent pour formuler et résoudre un problème d'environnement.

\section{Terrain et méthode de recherche : une étude de cas}

Confronter recommandations et pratiques consiste à identifier et mettre en regard les catégories de l'espace, du milieu et du territoire qui se trouvent mobilisées par les agriculteurs, avec celles des acteurs en charge de définir les recommandations environnementales. C'est ce que nous avons réalisé dans une petite région du Bassin parisien située aux environs de Migennes, dans l'Yonne. Dans le cadre d'une des premières OGAF-Environnement ${ }^{1}$, instaurée en 1993, une action préventive y a été mise en place autour de cinq captages d'alimentation en eau potable pollués par les nitrates. Ces captages alimentent en eau 6000 habitants des environs de Migennes, dans un paysage agricole dominé par les grandes cultures. Des périmètres ont été définis autour de ces captages pour délimiter les parcelles sur lesquelles les agriculteurs sont incités à changer certaines pratiques suivant des recommandations locales qui concernent des techniques culturales (installation de cultures pièges à nitrates) et l'ajustement de la fertilisation azotée en fonction de la méthode du bilan prévisionnel de l'azote. Dans cet article, les résultats s'appuient sur l'étude de la fertilisation azotée du blé, seule culture présente sur toutes les parcelles du

\footnotetext{
${ }^{1}$ Les opérations groupées d'aménagement foncier (OGAF) Environnement ont servi de support d'application du règlement agri-environnemental de la PAC. Dans un second temps, elles ont été également dénommées opérations locales agrienvironnementales (OLAE).
} 


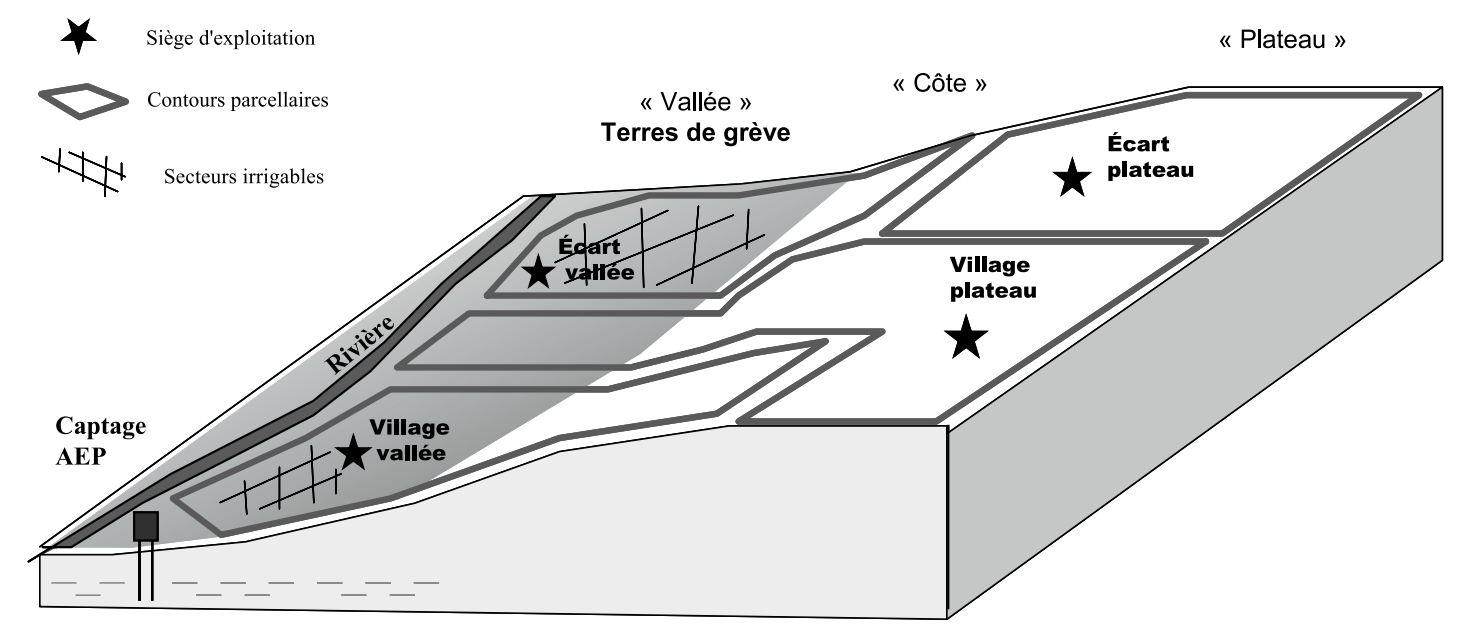

Fig. 1. Disposition des exploitations agricoles sur un versant type du Migennois.

\section{Encadré 1. Le dispositif d'enquêtes auprès des agriculteurs}

L'enquête combine des méthodes issues de la sociologie, de la géographie et de l'agronomie. Dans son principe général, elle repose sur une approche compréhensive des pratiques.

1. Caractériser des logiques pratiques. On cherche à identifier le raisonnement des pratiques, plus les conceptions qui sont en amont et qui leur donnent du sens. L'entretien se déroule pour l'essentiel à partir d'une discussion basée sur un support graphique représentant le plan d'exploitation dans son espace environnant. La discussion étant enregistrée, l'enquêteur inscrit sur ce support les informations que livre l'agriculteur : noms et qualitatifs de lieux, découpages de l'espace et toutes autres indications relatives à l'organisation spatiale des pratiques dans l'exploitation. Cette enquête comporte aussi un volet agronomique qui vise à replacer l'étude des pratiques techniques dans une compréhension de l'organisation générale de l'exploitation. Toutefois, même dans cette partie d'enquête, l'approche compréhensive est maintenue afin de privilégier le recueil du point de vue de l'agriculteur sur sa pratique.

2. Décrire et cartographier l'espace du travail de l'agriculteur. Les enquêtes décrites ci-dessus se fondent sur la mémoire de l'agriculteur qui restitue sa pratique. C'est la cohérence des arguments confrontés aux données de l'exploitation qui permet d'identifier le raisonnement de l'agriculteur, sa logique pratique, son comportement géographique. Pour analyser des pratiques spatiales, l'entretien doit être complété par une enquête qui se rapproche le plus possible de l'observation directe des pratiques « en acte». C'est ce que nous avons cherché à faire pour étudier les pratiques d'épandage d'engrais décrites sous forme de monographies de chantier. Une telle enquête exige d'être faite le jour même ou le lendemain de l'épandage. Cela permet de recueillir des informations géographiques à faible "durée de vie », tels l'ordre et la morphologie des trajets de l'épandeur d'engrais, les adaptations aux circonstances de la journée (circonstance climatique, vie familiale, travail), ou encore les imprévus et les erreurs qui surviennent et qui génèrent des réajustements de fumure inédits.

secteur d'étude. Pour l'étude des autres pratiques culturales, voir Soulard (1999).

Pour délimiter notre terrain d'observations, nous avons collecté et cartographié des informations sur les communes de l'OGAF, sur le milieu naturel défini par les études de circulation de l'eau et sur l'extension spatiale des exploitations agricoles du secteur. La figure 1 schématise l'organisation spatiale d'un versant type du secteur d'étude retenu, constitué par l'interfluve entre les rivières de l'Yonne, de l'Armançon et du Serein. À l'intérieur de cet espace, nous avons élaboré un protocole d'enquêtes dans les exploitations agricoles pour recueillir les discours et cartographier les informations se rapportant à l'espace de travail des agriculteurs (Encadré 1). Ce dispositif, établi dans le cadre strict de la réalisation d'une thèse (Soulard, 1999), a été enrichi par notre participation à une étude agronomique menée par l'INRA-SAD
(Morlon et al., 1996) et à une étude sociologique menée par le Gerdal et le Ladyss (Luginbühl et al., 1998).

\section{Les dimensions spatiotemporelles des recommandations techniques}

\section{L'espace et le temps des recommandations}

Dans l'OGAF du Migennois, les recommandations aux agriculteurs se fondent sur la transposition au niveau local des principes généraux du bilan prévisionnel de l'azote tel qu'il a été défini par les agronomes au niveau national (Encadré 2). Dans cette optique, les techniciens concernés par l'OGAF ont mis au point une politique de conseil fondée sur l'envoi régulier aux agriculteurs $\mathrm{d}^{\prime}$ « avertissements techniques». 


\begin{abstract}
Encadré 2. Le bilan prévisionnel de l'azote
Le bilan prévisionnel de l'azote sert à déterminer les apports d'engrais minéraux et organiques à apporter sur une culture (COMIFER, 1996).

Exprimé en unités d'azote pour une situation sol-culture donnée, le calcul du bilan consiste à estimer les besoins azotés de la culture, correspondant aux «sorties d'azote» (grains + pailles), auxquels on soustrait les « entrées d'azote " provenant à la fois des fournitures du sol (minéralisation de l'azote retenu dans la matière organique) et des apports azotés qu'effectue l'agriculteur (engrais minéraux et organiques). Le principe du bilan repose sur la recherche de l'équilibre «entrées-sorties » d'azote pour éviter, soit un appauvrissement du sol en azote (bilan négatif) pouvant causer une perte de rendement pour l'agriculteur, soit un excès d'azote dans le sol (bilan positif), cause potentielle de pollution par les nitrates.

Établi en vue de déterminer les apports d'azote sur une culture, ce bilan est prévisionnel. Il se base sur une estimation du rendement qu'il sera raisonnablement possible d'atteindre. Pour faire cette estimation, il est conseillé de se baser sur la moyenne des résultats obtenus les années précédentes (ex. : rendements réellement obtenus trois années sur cinq).
\end{abstract}

Ces avertissements donnent en référence aux agriculteurs des niveaux de fumure azotée à apporter sur leurs cultures en fonction du précédent cultural et du type de sol. Ils se présentent sous forme de références locales à moduler d'une année à l'autre selon les circonstances climatiques et les résultats d'analyses de l'azote minéral dans le sol à la sortie de l'hiver. Ils comprennent aussi des consignes de dates et de fractionnement des apports et sont pour cela envoyés aux agriculteurs juste avant les périodes d'épandage des engrais azotés minéraux.

Ainsi conçues et divulguées aux agriculteurs, les références temporelles qui structurent les recommandations sont le cycle végétatif de la culture. L'ajustement de la fertilisation consiste à combiner le temps long de l'historique cultural (effet précédent) et les moments-clés qui séquencent les décisions d'apports au cours du cycle cultural. La dimension spatiale est pour l'essentiel contenue dans la référence aux «types de sols » en fonction desquels plusieurs critères du bilan sont établis.

\section{La prise en compte des zones à risque de pollution}

Dans le Migennois, les sols de grève correspondent aux basses terrasses argilo-siliceuses des cours d'eau. Ils sont situés à la verticale des nappes alluviales qui alimentent les captages d'eau potable du secteur. Cette disposition dans l'espace leur confère une sensibilité particulière au risque de pollution des captages du secteur. Pour les hydrogéologues qui ont établi les périmètres de l'OGAF (SAFEGE, 1991), ces sols correspondent à une zone préférentielle d'infiltration de l'eau vers les nappes. Pour les agronomes qui ont été chargés d'évaluer les changements de pratiques (Morlon et al., 1996), ces sols sont sujets à de fortes variabilités, interannuelles et intraparcellaires, des rendements. Les rendements y sont difficiles à prévoir au moment recommandé pour appliquer la fertilisation. Aussi, les sols de grève correspondentils aux situations à risques pour lesquelles l'application du bilan prévisionnel de l'azote est un levier d'action insuffisant pour éliminer tout risque de fuite en nitrates sous des parcelles cultivées (Sebillotte et Meynard, 1990).

Identifiés par les spécialistes, les risques de pollution sur sols de grève n'ont pas été intégrés de la même manière par les conseillers et par les agriculteurs. Pour les conseillers, nos enquêtes révèlent que le choix des niveaux de rendements sur sols de grève a fait l'objet de négociations ardues entre les techniciens de la chambre d'agriculture et ceux des trois organismes fournisseurs d'engrais du secteur. Le compromis obtenu se traduit par un conseil de fumure supérieur à celui qu'aurait fourni l'application stricte des données du bilan avec une estimation plus réaliste des rendements potentiels. Du côté des agriculteurs, les "terres de grève » sont également identifiées. Selon eux, elles correspondent aux «mauvaises terres » du secteur, particulièrement sensibles au risque de sécheresse. Les agriculteurs associent ces terres à une multitude de qualificatifs généralement associés à la production ou au travail («terres séchantes", " faciles à travailler », « le blé grille», etc.) et rarement au risque de pollution.

Ainsi, nous constatons que les terres de grève sont un milieu spécifié par les différents acteurs en présence, mais avec des significations multiples. De part et d'autres, il y a convergence mais non équivalence des débats et jugements qui se rapportent aux terres de grève.

\section{Intérêts et limites des recommandations locales}

Les recommandations locales du Migennois comportent des limites évidentes, tout d'abord du fait même de leur conception, trop grossière et insuffisamment référée au risque de pollution. Il serait donc hasardeux de mener notre analyse en considérant ces recommandations comme la solution pertinente au problème posé.

Par contre, d'autres difficultés surgissent qui présentent un intérêt pour notre approche. Il s'agit des difficultés propres à l'application par les agriculteurs de la méthode du bilan prévisionnel de l'azote. Celles-ci sont connues depuis que les agronomes ont mené des enquêtes qui montrent que, même quand les agriculteurs 
connaissent les principes du bilan, ils sont peu nombreux à l'appliquer (Darré, 1983 ; Boiffin et al., 1984; Jeuffroy et al., 1987; Cerf et Meynard, 1988). Les raisons de ces difficultés ont été analysées sous plusieurs angles et nos enquêtes dans le Migennois corroborent les analyses qui ont été faites. Par exemple, Darré (1983) explique que les agriculteurs agrègent les critères agronomiques que les techniciens séparent, ils ne se réfèrent pas aux mêmes choses pour agir. De même, Aubry (1995) explique que les agriculteurs n'envisagent pas de prendre en compte la totalité des situations sols/cultures présentes sur leur exploitation. Ils privilégient quelques «parcelles-guides » qu'ils surveillent de près et en fonction desquelles ils déclenchent des dates d'apports d'engrais sur l'ensemble des parcelles d'une même culture. Tout semble donc indiquer que les agriculteurs ont une logique pratique fort différente de celle que présuppose le modèle technique. Or, le croisement entre fertilisation et risque de pollution nous livre une nouvelle piste d'analyse : celle du rapport à l'espace dans les pratiques.

En effet, nous avons vu que la prise en compte de l'environnement se matérialise essentiellement par la «mise en espace » des recommandations et des pratiques sur une catégorie de sols particulièrement sensible au risque de pollution. Nous pouvons dès lors faire l'hypothèse qu'une analyse spatialisée des pratiques de fertilisation apportera des éléments nouveaux. C'est donc ce que nous avons fait en examinant les ajustements que les agriculteurs opèrent au cours du temps, et dans l'espace, dans leurs pratiques concrètes allant de l'achat d'engrais aux apports azotés sur les parcelles.

\section{Les dimensions spatiotemporelles des pratiques de fertilisation}

L'analyse spatiotemporelle des pratiques de fertilisation nous conduit à identifier deux étapes fondamentales de l'ajustement, largement ignorées des recommandations techniques dans la mesure où la première intervient avant le début du cycle de culture (la commande d'engrais) et où la seconde décrit le travail que nécessite l'apport azoté lui-même (le chantier d'épandage sur les parcelles).

\section{Le premier ajustement au moment de la commande}

Si les recommandations se basent sur le cycle végétatif des cultures, dans l'exploitation agricole, l'ajustement azoté commence au moment de la commande d'engrais. Pour le blé, celle-ci est effectuée à l'automne (commande dite « morte saison »), c'est-à-dire longtemps avant l'apport azoté du printemps, parfois même avant le semis de la culture. Cette commande d'engrais repose sur une estimation des besoins annuels en engrais pour chaque culture et pour chaque élément fertilisant. Elle consiste à choisir les formules commerciales d'engrais au meilleur prix, dans la limite des formules disponibles chez les fournisseurs et dans des conditionnements adaptés à l'exploitation. Elle débouche sur des accords commerciaux qui prévoient des modalités de livraison et de consommation de la commande. Tout cela s'opère donc bien avant de connaître les besoins nutritifs des cultures. Or, cette antériorité de la commande est source «d'ajustements » multiples.

Premièrement, les écarts entre l'assolement prévu au moment de la commande et l'assolement réel étant fréquents, la commande d'un même engrais azoté pour plusieurs cultures peut être source de concurrences ou de couplages d'apports entre cultures ou parcelles différentes. Deuxièmement, le recours à des engrais complets (binaires ou ternaires) peut aussi générer des écarts à la prévision. Par exemple, sur conseil des fournisseurs, certains agriculteurs apportent des engrais complets « $\mathrm{N}$, $\mathrm{P}, \mathrm{K}$ » sur terres de grève. Selon les fumures commerciales disponibles, il peut arriver que le premier apport d'azote soit établi en fonction des fumures $\mathrm{P}$ et $\mathrm{K}$ nécessaires.

En fin de compte, la commande définit une « enveloppe globale d'engrais à répartir » entre parcelles et entre cultures. Elle se base sur des fumures moyennes par culture en fonction desquelles l'agriculteur évaluera ensuite le rythme d'utilisation de sa livraison d'engrais. Mais la commande peut être à l'origine d'écarts entre quantités achetées et nécessaires, générant ainsi des ajustements de fumure éloignés de ceux du bilan.

\section{Le deuxième ajustement lors du chantier d'épandage}

Le chantier d'épandage d'engrais sur les parcelles est un autre moment-clé de l'ajustement des apports suivant des critères qui relèvent de considérations pratiques. L'organisation de ce chantier est en effet le produit d'une combinaison de contraintes matérielles de plusieurs ordres, tels la performance de l'équipement d'épandage, la disponibilité en temps et en main d'œuvre au moment voulu, les trajets qu'impose la configuration spatiale du réseau de circulation et du parcellaire, auxquelles ils faut ajouter les circonstances d'intervention qui dépendent des caprices de la météorologie. Sans détailler ici la diversité des situations observables, nous mentionnerons deux faits majeurs qui conditionnent l'ajustement lors de la pratique d'épandage.

L'ajustement des apports azotés est tout d'abord guidé par le souci permanent qu'ont les agriculteurs de minimiser les risques d'erreur d'épandage (mettre moins ou plus). En effet, il n'est pas du tout évident pour 


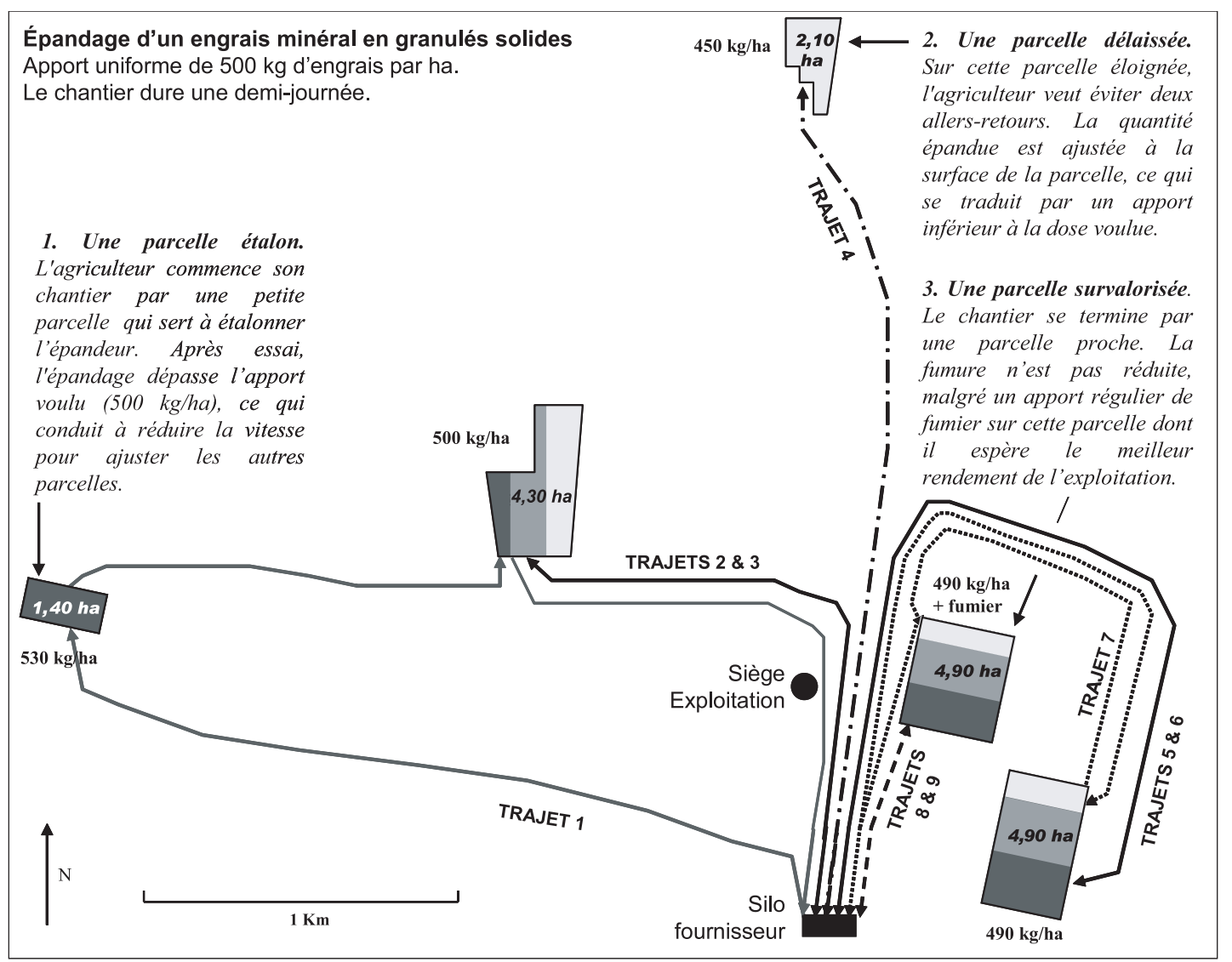

Fig. 2. Un chantier d'épandage d'engrais minéral.

l'agriculteur de connaître exactement la quantité qu'il épand sur ses parcelles. Il doit régulièrement étalonner son appareil - ne serait-ce que parce que la densité des engrais solides varie à chaque livraison - et il doit surveiller en permanence la régularité de l'épandage. Or, toute modulation des niveaux de fumure entre parcelles augmente les risques de se tromper, d'oublier de rectifier les réglages, et de perdre ainsi les repères du bon déroulement de l'épandage.

Mais l'ajustement est également conditionné par les exigences du transport de l'engrais, la circulation des engins, les opérations d'approvisionnement : bref, de tout ce qui constitue la logistique du chantier. En effet, l'épandage d'engrais est avant tout une pratique de transport de laquelle découle une recherche d'optimisation des trajets entre parcelles. C'est pourquoi les agriculteurs allotent les parcelles desservies à partir d'un même point en cherchant toujours à optimiser les déplacements. On observe ainsi des régularités dans les comportements spatiaux de différenciation des parcelles. Par exemple, les agriculteurs commencent l'épandage par des parcelles éloignées qui, en début de chantier, sont aussi celles où ils étalonnent le débit d'épandage. De même, ils utilisent une parcelle proche pour vider les restes du semoir avant réapprovisionnement ou garage (Fig. 2).

\section{Les différences de logiques d'ajustement entre exploitations agricoles et entre parcelles d'une même exploitation}

Au-delà des principes communs, les pratiques des agriculteurs s'opèrent suivant des modalités d'ajustement multiples. Nos enquêtes dans 20 exploitations du Migennois ont permis de dégager quelques «logiques d'ajustement » qui décrivent le lien entre une visée globale de l'agriculteur dans ses pratiques, les composantes matérielles de sa situation d'action et leurs conséquences sur l'organisation spatiotemporelle des apports d'azote sur les parcelles.

\section{À l'échelle des exploitations agricoles}

Sur cinq logiques d'agriculture identifiées, deux reposent sur des principes d'ajustement assez proches des recommandations du bilan.

La logique " globalisée par groupe de parcelles » repose sur la mise en œuvre de programmes de fertilisation établis pour des regroupements de parcelles obéissant à des critères relativement stables dans le temps. Ces regroupements varient entre exploitations : secteurs d'assolement; parcellaires irrigués; zones d'épandage des 
engrais de ferme; blocs de parcelles éloignées. Cette logique se rencontre dans les cinq exploitations agricoles les mieux dotées en moyens de production. Si la fertilisation azotée est différenciée par groupe de parcelles, le bilan prévisionnel de l'azote n'est pas vraiment utilisé pour déterminer les apports sur une campagne donnée. Il est davantage utilisé a posteriori pour évaluer les écarts entre fertilisation apportée et rendements obtenus. Le bilan prévisionnel s'intègre à une visée de maîtrise globale d'un système technique sans être pour autant un outil d'aide à la prise de décision.

Cette dernière observation différencie ce groupe du second dont la logique est « circonstancielle par parcelle». En effet, les trois agriculteurs de ce groupe recherchent des possibilités de prévoir et adapter le mieux possible les fumures azotées d'une parcelle à l'autre, d'un apport à l'autre. La notion de bilan prévisionnel est partie intégrante de leurs pratiques, mais en fonction de critères qui leur sont propres. Ces critères reposent sur la mémorisation d'états des parcelles qui matérialisent et synthétisent les interdépendances spatiotemporelles entre apports d'azote, rendements culturaux, données climatiques et fonctionnement du sol (ex. : couleur et tenue de la culture, composition et densité des repousses après récolte... ). Les recommandations du bilan sont une donnée parmi d'autres. Elles s'intègrent à un raisonnement empirique d'agriculteurs qui gèrent des exploitations de petite taille mais "bien tenues". Leur équipement est modeste mais soigné. Leur rapport au travail n'est pas tant fondé sur la maîtrise du temps et de l'espace que sur l'adaptation constante et l'apprentissage permanent.

Les trois autres logiques reposent sur des principes d'ajustement différents du bilan. Pour deux d'entre elles, soit 11 agriculteurs sur les 20, l'apport d'azote est tout simplement uniforme sur toutes les parcelles d'une même culture. Il faut distinguer deux cas de figures : dans 8 cas, cet apport est " uniforme sécuritaire ", c'està-dire établi en fonction d'un rendement moyen à atteindre, quelle que soit la parcelle; dans 3 cas, l'apport est « uniforme sous-optimal », car il s'agit avant tout de servir toute les parcelles en engrais en vue d'une production modeste de paille et de grains pour les animaux. À l'évidence, ces logiques correspondent aux pratiques les plus éloignées de celles que présupposent l'application du bilan. Le résultat important apporté par l'enquête est que cette situation ne peut pas être attribuée à une «mauvaise volonté » des agriculteurs en question. Elle est d'abord due au fait que ceux-ci sont toujours en train de parer au plus urgent. Ces agriculteurs ayant à gérer de grandes exploitations au parcellaire morcelé sans avoir la main-d'œuvre en conséquence, l'absence d'ajustement tient éminemment à des contraintes structurelles.

La cinquième et dernière logique se caractérise par une logique "conjoncturelle sur toute ou partie de la sole». Les trois agriculteurs concernés avaient auparavant des pratiques comparables au premier groupe, mais l'agrandissement des structures les a conduits à simplifier les techniques de production. D'une année à l'autre, cette simplification peut s'opérer de deux manières, soit via des apports uniformes, soit via des différenciations entre parcelles délaissées et parcelles soignées. Ici, l'ajustement dépend de critères économiques, favorables ou non à l'environnement.

\section{À l'échelle des parcelles de grève}

Pour aller plus loin dans l'analyse des pratiques et faire le lien avec le risque de pollution, nous avons voulu confronter les logiques pratiques des agriculteurs aux résultats effectivement obtenus sur les parcelles. Dans deux exploitations, nous avons pu réunir les données permettant de comparer les rendements visés, en fonction desquels sont définis les apports azotés, aux rendements obtenus que les agriculteurs ont calculés à partir des livraisons de récolte (comptage des bennes par parcelle récoltée). Les agriculteurs concernés appartiennent aux deux groupes dont les logiques d'ajustement sont les plus proches du bilan.

Les informations disponibles, à la parcelle pour l'exploitation I, et pour deux groupes de parcelles dans la II, permettent de voir comment les agriculteurs différencient leurs parcelles de grève, les plus sensibles au risque de pollution, des autres parcelles (Fig. 3). Tout d'abord, nous observons que les ajustements effectués sont limités. Dans les deux exploitations, la fourchette des rendements visés est inférieure à celle des rendements obtenus : respectivement $27 \mathrm{q}$ et $52 \mathrm{q}$ par parcelle pour I; 20 et $30 \mathrm{q}$ par groupe de parcelles pour II. Deuxièmement, les ajustements ne s'opèrent pas avec la même approche dans les deux exploitations.

Dans ses terres de grève, l'exploitant I (Fig. 3a) se base en première approche sur un rendement maximum, établi en fonction de ce qui est obtenu réellement une année sur six. Lorsqu'il ajuste ce niveau de départ en fonction des circonstances de l'année, il effectue une révision à la baisse. Cette révision s'élève à 10 quintaux maximum alors que les rendements peuvent descendre à $30 \mathrm{q}$ de moins que le niveau visé initialement. Interrogé sur ce point, l'agriculteur répond qu'il ne peut pas se permettre de se baser en deçà d'un seuil de 60 q de blé qu'il considère comme le seuil minimum rentable. Il raisonne donc sa fertilisation en fonction d'un objectif de rendement atteint les bonnes années. A contrario, il ne raisonne pas de cette façon sur ses meilleures terres. Même si les rendements obtenus dépassent ici les rendements visés, l'agriculteur ne cherche pas à réévaluer ses fumures, comptant alors, peut-être inconsciemment, sur les restitutions d'azote du sol. S'il applique correctement la démarche du bilan sur ses meilleures terres, sur 
(a) Exploitation I : rendements visés et obtenus sur 19 parcelles sur six campagnes

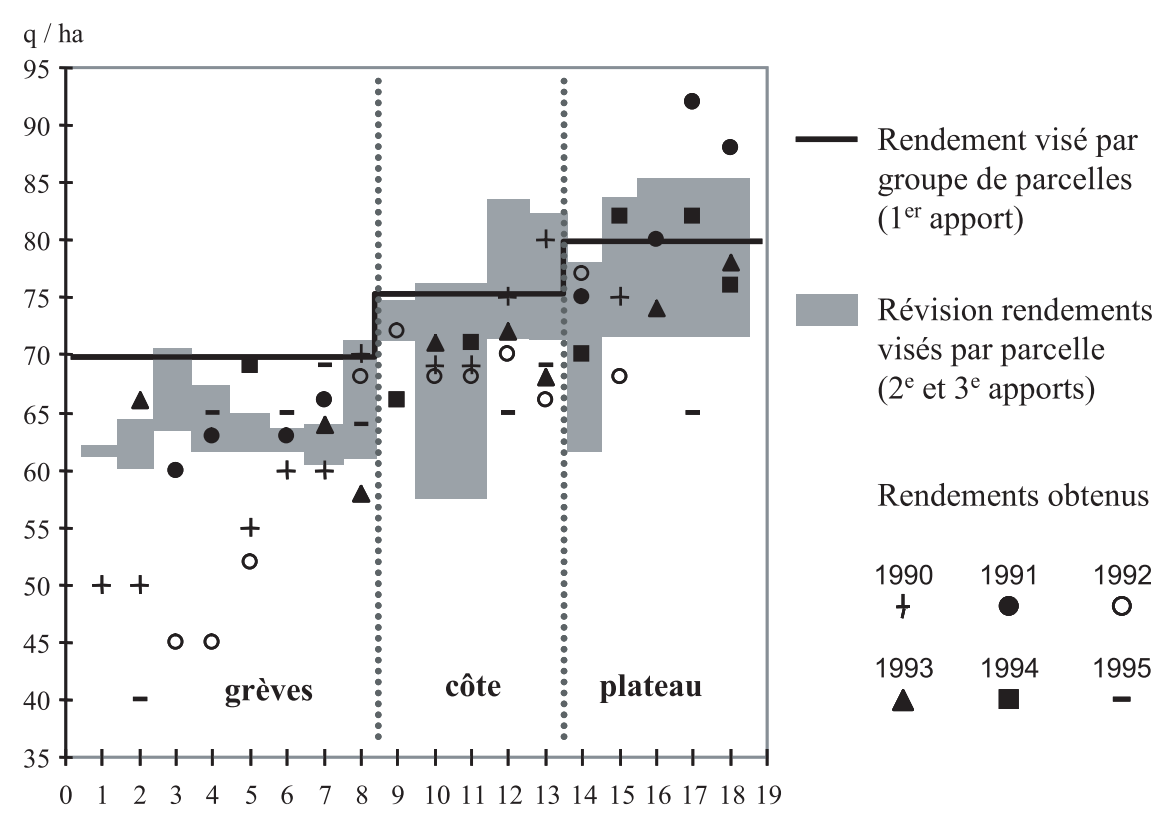

Parcelles classées par ordre croissant des rendements

(b) Exploitation II : rendements visés et obtenus par groupe de parcelles en 10 ans

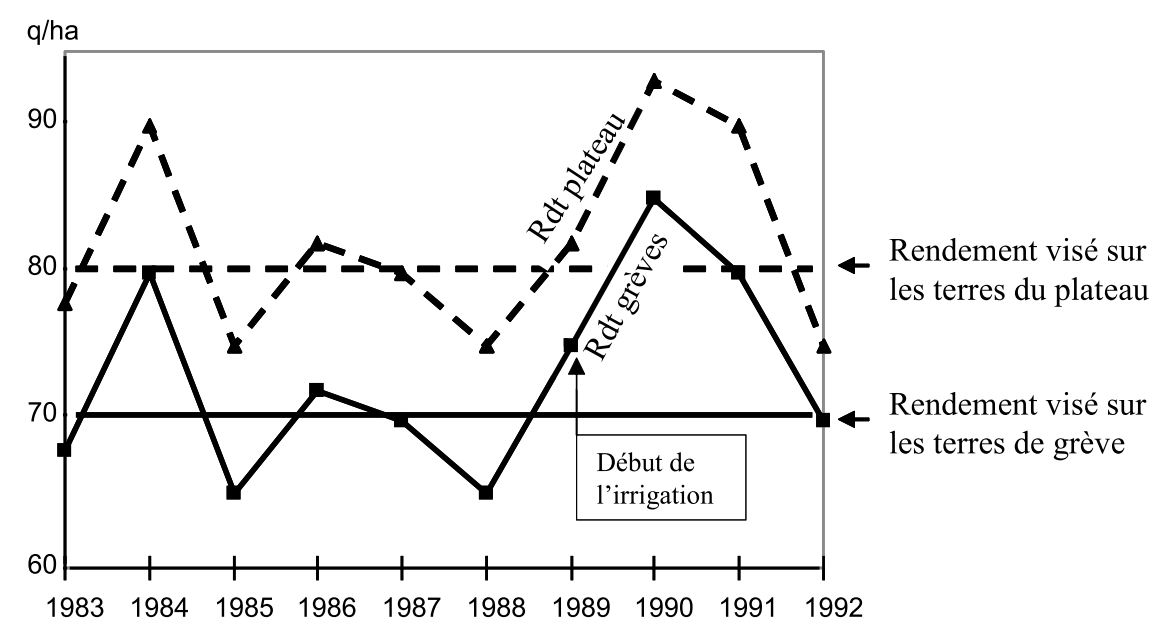

Fig. 3. Confrontation des rendements visés et obtenus dans deux exploitations.

terres de grève, il vise un rendement qu'il arrive à obtenir effectivement une année sur six.

L'exploitant II (Fig. 3b) dispose d'informations sur les rendements moyens obtenus en blé sur deux groupes de parcelles : les terres de grève et les «bonnes terres » du plateau. Sur dix ans, les rendements visés dans les deux catégories de terre correspondent bien à la moyenne des rendements moyens obtenus. L'introduction de l'irrigation de complément réduit l'écart entre le plateau et les grèves (il passe de -20 à -10 q par an), alors que le niveau de rendement visé reste le même. Elle se traduit donc par une meilleure adéquation entre rendements visé et obtenu. On peut ainsi dire, a priori, que la pratique de l'agriculteur II présente moins de risque que celle de I, puisqu'il vise comme rendement ce qu'il obtient effectivement en moyenne sur 10 ans.

\section{De l'analyse des pratiques au diagnostic agri-environnemental}

\section{Évaluer les écarts entre recommandations et pratiques}

Nos observations montrent que les agriculteurs pensent et organisent leurs pratiques suivant des catégories spatiotemporelles spécifiques. 
Premièrement, les agriculteurs se réfèrent à des catégories d'espace et de temps différentes de celles du bilan. $\mathrm{Au}$ plan temporel, tandis que les recommandations se fondent sur le cycle végétatif des cultures, les agriculteurs articulent des "campagnes" (prévision annuelle des fumures) avec des «saisons » (commandes et stockages d'engrais pour des groupes de cultures d'hiver et de printemps) et des «chantiers » (épandage d'engrais sur des parcelles à servir à une même date). Au plan spatial, le couple sol-culture des recommandations n'est qu'une composante, localement mineure, des critères suivant lesquels les agriculteurs différencient les apports azotés. Par endroits, les impératifs d'organisation logistique des chantiers peuvent empêcher la prise en considération des critères du bilan, en particulier dans les situations où les objectifs d'optimisation des trajets et d'application du bilan entrent en contradiction. C'est le cas des parcelles éloignées, dispersées ou peu accessibles.

Deuxièmement, même quand les recommandations visent des ensembles spatiaux bien identifiés par les agriculteurs, ces derniers ne différencient pas leurs pratiques en fonction du risque de pollution. Nous avons observé ce cas de figure au niveau des terres de grève. Si ces terres sont bien une catégorie du milieu que les agriculteurs connaissent et prennent déjà en compte dans leurs pratiques, les ajustements de fertilisation azotée qu'ils y effectuent sont largement inférieurs à ce que supposerait une prise en compte effective du risque de pollution.

Pour comprendre comment les agriculteurs peuvent se saisir du risque de pollution, comme dans les terres de grève du Migennois, nos résultats nous conduisent à proposer une mise en perspective territoriale de l'analyse.

\section{Relier pratiques localisées et risques de pollution sur terres de grève}

Pour réaliser ce diagnostic, nous avons resitué l'étude des pratiques de fertilisation dans une analyse globale des modes de mise en valeur des terres de grève dans les exploitations du secteur d'étude. Ceci a consisté à mener une description localisée des pratiques agricoles sur parcelles de grève qui croise deux unités d'observations, l'exploitation agricole et l'unité de sol. Les principaux résultats de cette étude, exposés en détail dans Soulard (1999), font ressortir la coexistence sur terres de grève de deux systèmes de culture (au sens de «manières de cultiver $»)$.

Le premier système de culture est observé sur les parcelles irrigables où se succèdent des cultures d'hiver (céréales, colza) et des cultures de printemps (pois, maïs, betteraves, orge de brasserie). L'irrigation permet d'y obtenir des rendements se rapprochant de ceux obtenus sur les bonnes terres. Pour y arriver, les agriculteurs cherchent à ajuster au mieux les intrants. Sur le blé, la fertilisation azotée est fractionnée en trois fois, voire en quatre, et l'irrigation de complément est intégrée au raisonnement d'ajustement des apports. Dans le territoire, ce mode de mise en valeur des terres de grève est caractéristique des exploitations d'écart (Fig. 1) qui ont introduit l'irrigation dès les années 1960, ce qui leur permet d'avoir une succession culturale à base de betteraves sucrières pratiquée sur l'ensemble du parcellaire exploité. Ce système de culture est aussi présent dans les exploitations des villages des vallées qui ont réussi à constituer un ou deux groupes de parcelles irrigables en vue de réduire les risques de sécheresse et d'introduire le maïs grain (ces exploitations n'ont pas de betteraves). Dans ces deux types d'exploitation, la conduite technique des parcelles de grève étant délicate, les agriculteurs cherchent à ajuster la fertilisation, mais de façon trop limitée. Le risque de pollution provient d'un degré d'ajustement des pratiques aux parcelles insuffisant.

Le second système de culture est observé sur les autres parcelles de grève. De surfaces hétérogènes, très dispersées et fréquemment enserrées dans des bosquets, ces parcelles sont réputées de faible valeur pour la culture. Les agriculteurs y ont remplacé l'ancienne pratique alternant cultures de céréales et jachères (appelées «sombres »), pâturées ensuite par des ovins, par des cultures annuelles conduites de façon simplifiée, voire par la concession ou la vente de ces terres pour l'extraction de gravier. Les combinaisons culturales observées vont de la succession de cultures d'hiver (colza, blé, orge) au gel des terres (gel fixe de la PAC), en passant par la monoculture d'orge. Ce mode de mise en valeur est caractéristique des exploitations des villages du plateau, à dominante humide, pour lesquelles les terres de grève combinent deux handicaps, liés à leur faible valeur productive et à l'éloignement, mais représentent aussi une sécurité de production en année humide. Ce système de culture est aussi présent dans les parties non irrigables des exploitations des villages de vallée pour qui ces parcelles symbolisent à la fois la fragilité productive de l'exploitation et les promesses financières d'un patrimoine foncier convoité pour l'extraction de gravier. Dans ces parcelles, l'ajustement de la fertilisation azotée est motivé par un souci d'économie et de simplification du travail. Il est soit absent, soit opéré par défaut (parcelles délaissées). Le risque de pollution provient alors de pratiques irrégulières et peu suivies.

\section{Combiner innovation technique et gestion de l'espace}

L'analyse des pratiques sur terres de grève met en évidence des caractéristiques spatiales qu'il conviendrait d'intégrer dans un raisonnement environnemental local.

Une première caractéristique tient à la dualité agricole des terres de grève, matérialisée par le caractère irrigable ou non des parcelles. À ce niveau, l'analyse des leviers 
d'action devrait s'appuyer sur la gestion par les agriculteurs de la répartition spatiotemporelle du risque de sécheresse sur leur exploitation. C'est en effet en fonction de l'appréhension de ce risque que l'on pourra comprendre les marges de manœuvre dont ils disposent, soit pour assumer une gestion extensive de parcelles peu productives, soit pour, au contraire, accéder à une technicité supérieure, permettant de concilier risque de pollution et conduite de parcelles irriguées à fort potentiel de production.

Une seconde caractéristique tient aux multi-usages des terres de grève, où coexistent plusieurs systèmes de culture, plusieurs interventions sur la ressource en eau (irrigation, eau potable) et des usages non agricoles de l'espace cultivé (pour l'extraction de gravier, le dépôt de déchets divers, l'évacuation des eaux usées...). Or, ces différents usages constituent autant de pressions potentielles sur l'environnement. Apparemment disjoints, ils interfèrent avec les stratégies productives des agriculteurs, qu'il faudrait donc aussi analyser de ce point de vue. Cette analyse permettrait alors d'ouvrir une perspective d'action plus ambitieuse et efficace, en combinant innovation technique agricole et action foncière, en associant aux changements de pratiques agricoles un projet de mise en cohérence territoriale des usages sur terres de grève. Coupler innovation technique et gestion de l'espace serait une voie prometteuse pour intégrer localement le problème de pollution à un projet de développement durable.

\section{Conclusion : pour une géographie des pratiques}

Tout au long de cette recherche, notre fil conducteur a été $\mathrm{d}$ 'analyser les relations des agriculteurs au problème de la pollution. Pour des raisons de méthode, nous avons choisi d'aborder cette étude indirectement, par l'analyse des écarts entre recommandations techniques et logiques pratiques. Nous avons privilégié l'étude de la dimension spatiale de ces écarts, ce qui nous a conduit à repérer des catégories spatiales qui émergent et se confrontent dans le traitement local d'un problème d'environnement.

Par ce travail, nous avons voulu spécifier un point de vue possible de la géographie sur l'analyse d'un problème d'environnement. Celui-ci est à la fois pluridisciplinaire et spécifique. Par la méthode d'étude des conceptions et des pratiques des agriculteurs, il emprunte à la sociologie des connaissances et de l'action. Mais il s'en distingue bien, car l'objet de recherche est le repérage de ce qui, dans des faits sociaux observés, tient à la disposition dans l'espace, le territoire ou le milieu, d'objets matériels porteurs de significations sociales particulières : les catégories de terres, les distances et circuits d'accès aux parcelles, les lieux d'implantation des captages, etc. Notre point de vue se rapproche aussi de l'agronomie, tout en s'en distinguant, car notre objectif n'est pas l'étude du fonctionnement du champ, du système de culture ou du système de production considérés en tant que tels. Notre objet est le repérage d'associations privilégiées entre des faits techniques localisés et des configurations territoriales particulières, tels les formes spatiales des exploitations ou les regroupements de parcelles opérationnels et, plus généralement, ce qui définit et configure l'espace du travail des agriculteurs.

\section{Remerciements}

L'auteur remercie François Papy, Jean-Pierre Deffontaines et Nicole Mathieu pour leur soutien, ainsi que les relecteurs de cet article.

\section{Références}

Aubry, C., 1995. Gestion de la sole d'une culture dans l'exploitation agricole. Cas du blé d'hiver en grande culture dans la région picarde. Thèse, INA P-G, Paris.

Bertrand, G. et C., 2002. Une géographie traversière : l'environnement à travers territoires et temporalités, Paris, Arguments.

Blanc-Pamard, C., 1986. Dialoguer avec le paysage ou comment l'espace écologique est vu et pratiqué par les communautés rurales des hautes terres malgaches, in Chatelin, Y., Riou, G. (Eds), Milieux et paysages, Paris, Masson, 17-36.

Boiffin, J., Bourgeois, A., Lépine, M., 1984. Les obstacles au développement de la fertilisation raisonnée, in COMIFER, Premier forum national de la fertilisation, Paris, 21-22 janvier 1982, Paris, ACTA, 7-17.

Cerf, M., Meynard, J.-M., 1988. Enquête sur la mise en œuvre de la fertilisation raisonnée, in COMIFER, Troisième forum national de la fertilisation raisonnée, Nancy, 22-23 juin 1988, Paris, COMIFER, 5-12.

COMIFER, 1996. Calcul de la fertilisation azotée des cultures annuelles : guide méthodologique pour l'établissement des prescriptions locales. Rapport.

Darré, J.-P., 1983. La Culture du blé: systèmes de pensée et élaboration des normes dans les groupes locaux. Rapport CAESAR, Paris.

Deffontaines, J.-P., Brossier, J., 2000. Système agraire et qualité de l'eau. Efficacité d'un concept et construction négociée d'une recherche, Natures Sciences Sociétés, 8, 1, 14-25.

Jeuffroy, M.-C., Meynard, J.-M., Sevat, B., 1987. Identification des obstacles à la mise en œuvre des méthodes de fertilisation vulgarisées, Bull. Tech. Inf., 295, 851-854.

Jollivet, M., Pavé, A., 1993. L'environnement : un champ de recherches en formation, Natures Sciences Sociétés, 1, 1, 6-20.

Luginbühl, Y., Leguen, R., Lemery, B., Sigwalt, A., Soulard, C., Toublanc, M., 1998. Écologie et nouveaux modes de gestion du territoire. Contrat ministère de l’Environnement 94-169. 
Mathieu, N., 1992. Géographie et interdisciplinarité : rapport naturel ou rapport interdit?, in Jollivet, M. (Ed.), Sciences de la nature, sciences de la société : les passeurs de frontières, Paris, CNRS Éditions, 129-154.

Morlon, P., Soulard, C., Maigrot, J.-L., Trouche, G., 1996. Comment traiter un problème de pollution par les nitrates? Évaluation de l'OGAF du Migennois. Rapport INRA, Dijon.

Papy, F., 2001. Pour une théorie du ménage des champs : l'agronomie des territoires, C.R. Acad. Agric. Fr., 87, 4, 139-149.

Pinchemel, P., Pinchemel, G., 1995 (rééd). La Face de la terre, Paris, Armand Colin.

Rémy, É., November, V., D’Alessandro-Scarpari, C., Charvolin, F. (Eds), 2004. Espaces, savoirs et incertitudes, Paris, Ibis Press.
Robic, M.-C., Mathieu, N., 2001. Géographie et durabilité : redéployer une expérience et mobiliser de nouveaux savoirfaire, in Jollivet, M. (Ed.), Le Développement durable, de l'utopie au concept, Paris, Elsevier SAS, 67-190.

SAFEGE, 1991. Détermination des bassins d'alimentation de cinq captages AEP du Migennois. Première phase. Rapport d'étude.

Sebillotte, M., Meynard, J.-M., 1990. Systèmes de culture, systèmes d'élevage et pollutions azotées, in Calvet, R. (Ed.), Nitrates, agriculture, eau, Paris, INRA, 289-312.

Soulard, C.T., 1999. Les Agriculteurs et la pollution des eaux. Proposition d'une géographie des pratiques. Thèse de doctorat, Université Paris I - Panthéon Sorbonne, Paris.

Reçu le 29 avril 2004. Accepté le 3 mars 2005.

To access this journal online: www.edpsciences.org 